\title{
Nanophotonics
}

Diamondoid Molecules: with Applications in Biomedicine, Materials Science, Nanotechnology \& Petroleum Science

Ognjen Perišić 


\title{
BOOK REVIEW
}

\section{Diamondoid Molecules: with Applications in Biomedicine, Materials Science, Nanotechnology \& Petroleum Science}

\author{
G. A. Mansoori, P. Lopes Barraos de Araujo and E. Silvano de Araujo, Eds., 400+ pages, ISBN \\ 10: 9814291609, ISBN 13: 978-9814291606; Edition: 1; World Scientific Publishing Co., \\ Hackensack, NJ (2012), \$128 hardcover, \$166 e-book. \\ Reviewed by Ognjen Perišić, Vojvode Brane 32, 11000 Belgrade, Serbia, Phone: +381 65544 \\ 7919, ognjen.perisic@gmail.com
}

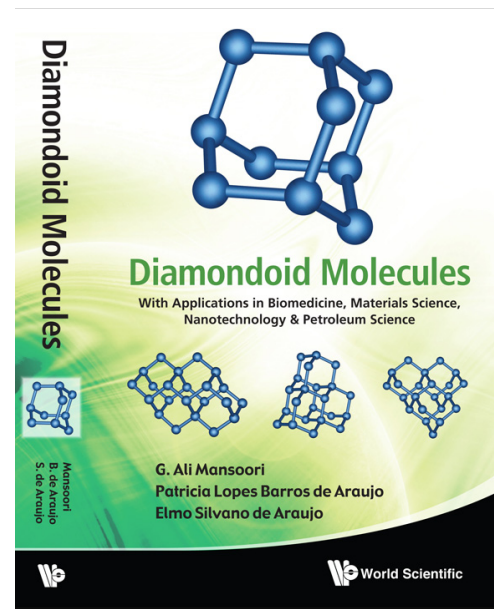

The pharmaceutical and biotech industries face difficult problems. Namely, enormous amounts of capital are spent on research and development, but a very small number of tested compounds become truly marketable. Moreover, many of the compounds currently on the market are, in essence, evolutionary designed. Thus, although it is equipped with the most advanced technology and backed up with a comprehensive theoretical framework, the current biotech industry lags in productivity when compared to the old-style, animal-model-based research. Some researchers have expressed their belief in a need for a paradigm shift. But what might that paradigm shift be? It may be a new way to screen and test molecules, but it could also be an introduction of a completely new set of molecular building blocks. The new book by G. A. Mansoori et al. on diamondoid molecules may be one of the first steps toward that shift. Diamondoid Molecules is a thorough presentation of current and future applications of diamondoids. It is composed of seven chapters and a comprehensive glossary. Every chapter is excellently illustrated with plenty of clearly designed figures and graphs and well-organized tables.

The book shows that diamondoids are molecules with many applications in materials science, as well as in petroleum science and technology, and demonstrates their great potential for derivatization. Diamondoids are seen as important molecular building blocks in biomedicine and bottom-up nanotechnology. They are expected to become crucial design elements of nanoelectromechanical (NEMS) and microelectromechanical systems (MEMS), and new medicines. They are already used in the design of drugs for fighting infectious diseases such as influenza, HIV, tuberculosis (Mycobacterium tuberculosis) and various other bacterial (Gram-positive bacteria) and parasitic infections. Furthermore, it is anticipated that cancer will be efficiently fought with diamondoids' derivatives (cancer chemotherapy and anticancer drugs). They can also act as neuroprotective agents for Alzheimer`s disease, as well as drugs with antidotal effects or hypoglycemic action, or drugs for diabetes treatment. Future applications of diamondoids may be nanodrug delivery and drug nanotargeting.

In addition to giving a good overview of diamondoids' role in biomedicine and nanotechnology, the book is an excellent introduction to theoretical and technological aspects behind diamondoids' chemistry, their molecular structure and physical properties. The emphasis is given to quantum calculations of diamondoids' molecular properties. It is shown that ab-initio calculations are quite useful for the prediction of these properties, especially in light of the huge number of diamondoid molecules and their derivatives in existence. The book gives an excellent overview of quantum confinement effects and negative electron affinities of diamondoids, a unique characteristic of these molecules. It also describes the first-principles simulations of 
diamondoids' interactions with other nanoscale systems, such as carbon nanotubes, metallic surfaces, and metallic atomic force microscope cantilever tips. This is an important part of the whole manuscript because those interactions are promising pathways toward an efficient design of new nanomaterials. Additionally, the book gives a good presentation of functionalized diamondoids, molecules instrumental in the creation of many nanosystems with predetermined chemical and physical properties, already applied in pharmacology and nanoelectronic devices. A careful reader of this book will learn that diamondoids are generally optically transparent in visible light and have high electrical insulating properties, as diamonds generally have. It can be grasped that many diamondoid derivatives have properties with potentially very interesting optoelectronic applications.

Therefore, I highly recommend Diamondoid Molecules to students of bioengineering, nanotechnology, and material and petroleum science, as well as to professional researchers. 